\title{
Excitation of edge magnetoplasmons in semi-infinite graphene sheets: Temperature effects
}

\author{
Weihua Wang, ${ }^{*}$ Jari M. Kinaret, and S. Peter Apell \\ Department of Applied Physics, Chalmers University of Technology, SE-412 96 Göteborg, Sweden
}

(Received 28 October 2011; revised manuscript received 11 June 2012; published 21 June 2012)

\begin{abstract}
We investigate edge magnetoplasmons in semi-infinite graphene sheets. Similar to an ordinary two-dimensional electron system (2DES), at zero temperature graphene sheets exhibit two magnetoplasmon branches, a higherenergy one starting at the $n=1$ Landau level (cyclotron frequency) and a lower-energy edge branch starting at zero frequency. However, in contrast to a 2DES with parabolic dispersion, at nonzero temperatures an extra magnetoplasmon branch arises at the $n=1$ Landau level and has the same edge current rotation as the lowerenergy branch.
\end{abstract}

DOI: 10.1103/PhysRevB.85.235444

PACS number(s): 73.20.Mf, 71.35.Ji, 78.67.Wj

\section{INTRODUCTION}

Graphene is a one-atom-thick two-dimensional (2D) carbon crystal that is the basic building block for all graphitic materials. Electrons and holes in graphene are described by an effective Dirac equation with a zero mass. This special energy spectrum leads to several unusual transport properties that have been intensively studied both experimentally and theoretically. ${ }^{1-3}$ Graphene has novel electromagnetic properties as well. The universal optical conductance of graphene is governed by the fine structure constant and is independent of wavelength over a broad range. ${ }^{4}$ In a specific frequency window, graphene supports a weakly damped TE surface mode, ${ }^{5,6}$ which has been used to design a broadband TE-pass polarizer. ${ }^{7}$ Controlling the electron chemical potential in graphene by a gate voltage, Liu et al. ${ }^{8}$ have reported a graphene-based electroabsorption modulator, and Vakil and Engheta ${ }^{9}$ proposed to build infrared metamaterials and transformation optical devices. Especially, atomically localized plasmons have been observed in monolayer graphene, ${ }^{10}$ which could have potential application in integrated optics.

In finite graphene systems, like $p$ - $n$ junctions ${ }^{11}$ and ribbons or disks, ${ }^{12,13}$ plasmon excitations are easily tailored by gate voltages and, moreover, have lower losses ${ }^{14}$ than in a metal. Using periodic graphene ribbons or disks, a high efficiency optical excitation of the plasmons can be achieved ${ }^{8,15-17}$ and, due to the unusual electronic spectrum, these plasmons can exist at very low frequencies such as the THz range. This can be used in graphene-based THz optically pumped lasers. ${ }^{18,19}$ All this makes graphene a promising platform for nanoplasmonics.

\section{EDGE MAGNETOPLASMONS IN SEMI-INFINITE SHEETS}

Along the boundary of a semi-infinite conducting sheet $(x<0)$, gapless edge magnetoplasmons can be excited in a perpendicular magnetic field, ${ }^{20-23}$ and they do not need to overcome the energy gap of single-particle excitations. At the relatively low-frequency quantum Hall regime, the edge magnetoplasmons have been studied extensively in a twodimensional electron system $(2 \mathrm{DES})^{24-28}$ and very recently also in armchair graphene ribbons. ${ }^{29,30}$ The optical response of magnetoplasmons in graphene has been experimentally studied both in patterned and unpatterned systems. ${ }^{31,32}$ In this article, we study the high-frequency edge magnetoplasmon excitations in semi-infinite graphene sheets. The in-plane potential $\Phi(x, y, t)=\exp [i(q y-\omega t)] \Phi(x)$ and related chargedensity oscillation can be obtained self-consistently ${ }^{33,34}$ from the Poisson equation: ${ }^{35}$

$$
\Phi(x)=\frac{1}{\epsilon} \int_{-\infty}^{0} d x^{\prime} L_{q}\left(x-x^{\prime}\right)\left[\rho\left(x^{\prime}\right)+\rho^{*}\left(x^{\prime}\right)\right],
$$

where the integral kernel is given by $L_{q}\left(x-x^{\prime}\right)=K_{0}(q \mid x-$ $\left.x^{\prime} \mid\right) / 2 \pi .^{22,23,34}$ Here $\epsilon$ is the average surrounding dielectric constant, for instance, with $\mathrm{SiO}_{2}$ as the substrate, $\epsilon=\epsilon_{r} \epsilon_{0}=$ $\left(\epsilon_{\mathrm{vac}}+\epsilon_{\mathrm{sub}}\right) / 2=2.5 \epsilon_{0}$. The two-dimensional (2D) sheet and edge charge densities, $\rho(x)$ and $\rho^{*}(x)$, are related to the potential through

$$
\begin{aligned}
\rho(x) & =\frac{\sigma_{x x}(\omega)}{i \omega}\left[q^{2}-\partial_{x}^{2}\right] \Phi(x), \\
\rho^{*}(x) & =\frac{\delta(x)}{i \omega}\left[\sigma_{x x}(\omega) \partial_{x} \pm i \sigma_{x y}(\omega) q\right] \Phi(x) .
\end{aligned}
$$

Here, $q$ and $\omega$ are defined to be positive and \pm indicates edge currents flowing in different directions relative to the magnetic field, and $\delta(x)$ arises from the step function in the conductivity tensor at the edge of the graphene sheets. ${ }^{23,33}$

Equation (1) can be solved analytically, if the exact integral kernel is approximated by a simpler one $L_{0}(x)=2^{(-3 / 2)} \exp (-\sqrt{2} q|x|)$ [with equal area and second momentum $^{20-23}$ ], and can be decoupled into two local differential equations for $x<0$ and $>0$, respectively:

$$
\begin{aligned}
& \left(\partial_{x}^{2}-2 q^{2}\right) \Phi_{-}(x)=-\frac{q}{\epsilon} \rho(x), \quad x<0, \\
& \left(\partial_{x}^{2}-2 q^{2}\right) \Phi_{+}(x)=0, \quad x>0 .
\end{aligned}
$$

Defining the auxiliary functions

$$
\begin{aligned}
& \eta=\frac{q}{i \epsilon \omega} \sigma_{x x}, \\
& \chi=\frac{q}{\epsilon \omega} \sigma_{x y},
\end{aligned}
$$

combining Eqs. (2)-(4), and requiring that the exponentially growing components are absent yields the implicit dispersion relation $\eta^{2}-\chi^{2}-3 \eta \pm 2 \sqrt{2} \chi=0 .^{20-23}$ The exponential approximation for the kernel leads to an underestimation of the plasmon frequencies at $q \rightarrow 0$-for instance, the upper branch starts below the cyclotron frequency-which can be 
remedied by slightly changing the coefficient of $\chi$ from $2 \sqrt{2}$ to 3 (this also gives a good agreement with the numerical solution using the full kernel), resulting in the approximate dispersion relation:

$$
\eta^{2}-\chi^{2}-3 \eta \pm 3 \chi=0 .
$$

All material dependent properties of the plasmon excitations arise from the conductivity tensor $\sigma$, which is fundamentally different for graphene with a Dirac-like spectrum as opposed to the conventional 2DES with the parabolic dispersion relation; below, we will compare the analytic solution Eq. (5) for the approximate kernel with a numerical solution using the full kernel and show that the approximation is well justified.

\section{EDGE MAGNETOPLASMONS IN GRAPHENE}

The bulk magnetoplasmon excitations have been theoretically studied. . $^{36-38}$ The peculiar properties are the unique nature of the Landau levels in graphene, as we will see below, which also essentially govern the features of the edge magnetoplasmon excitations.

\section{A. Magneto-optical conductivities of graphene}

In a perpendicular magnetic field, Landau levels in graphene are not equally spaced ${ }^{39}$ but are given by

$$
E_{n}= \pm \sqrt{n} \hbar \omega_{B},
$$

where $\omega_{B}=\sqrt{2} v_{F} / l_{B}$, and the \pm represent an electronlike $(+)$ or holelike (-) Landau level index. Here $v_{F}=10^{6} \mathrm{~m} / \mathrm{s}$ is the Fermi velocity in graphene and $l_{B}=\sqrt{\hbar / e B}$ is the magnetic length. Crucial to the following discussion, the $n=0$ Landau levels for electrons and holes are degenerate with $E_{0}=0$; i.e., their energy coincides with the energy at the Dirac point in the absence of a magnetic field.

The frequency dependent conductivity tensor for graphene can be obtained from linear-response theory. In a constant scattering time approximation, it is given by ${ }^{40}$

$$
\begin{aligned}
\sigma_{x x}(q=0, \omega)= & \frac{e^{2}}{2 \pi \hbar} \sum_{n=0}^{\infty}\left\{i \omega_{B}\left(\omega+i \tau^{-1}\right) \frac{\left[n_{F}\left(E_{n}\right)-n_{F}\left(E_{n+1}\right)\right]+\left[n_{F}\left(-E_{n+1}\right)-n_{F}\left(-E_{n}\right)\right]}{\left[\left(\omega+i \tau^{-1}\right)^{2}-f_{\text {intra }}^{2}(n) \omega_{B}^{2}\right] f_{\text {intra }}(n)}\right. \\
& \left.+i \omega_{B}\left(\omega+i \tau^{-1}\right) \frac{\left[n_{F}\left(-E_{n}\right)-n_{F}\left(E_{n+1}\right)\right]+\left[n_{F}\left(-E_{n+1}\right)-n_{F}\left(E_{n}\right)\right]}{\left[\left(\omega+i \tau^{-1}\right)^{2}-f_{\text {inter }}^{2}(n) \omega_{B}^{2}\right] f_{\text {inter }}(n)}\right\}, \\
\sigma_{x y}(q=0, \omega)= & \frac{e^{2}}{2 \pi \hbar} \sum_{n=0}^{\infty}\left\{\left[n_{F}\left(E_{n}\right)-n_{F}\left(E_{n+1}\right)\right]-\left[n_{F}\left(-E_{n+1}\right)-n_{F}\left(-E_{n}\right)\right]\right\} \\
& \times\left\{\frac{\omega_{B}^{2}}{\left(\omega+i \tau^{-1}\right)^{2}-f_{\text {intra }}^{2}(n) \omega_{B}^{2}}+\frac{\omega_{B}^{2}}{\left(\omega+i \tau^{-1}\right)^{2}-f_{\text {inter }}^{2}(n) \omega_{B}^{2}}\right\},
\end{aligned}
$$

where $n_{F}\left(E_{n}\right)=1 /\left\{1+\exp \left[\left(E_{n}-\mu_{c}\right) / k_{B} T\right]\right\}$ is the FermiDirac distribution, $\mu_{c}$ is the chemical potential, and

$$
\begin{aligned}
& f_{\text {intra }}(n)=\sqrt{n+1}-\sqrt{n}, \\
& f_{\text {inter }}(n)=\sqrt{n+1}+\sqrt{n} .
\end{aligned}
$$

These two functions determine the single-particle excitation spectrum of intra- and interband transitions, and particularly they are the same for $n=0$. Intrinsic relaxation time ${ }^{13}$ $\tau=\mu \mu_{c} / e v_{F}^{2}$ is used in our calculations, where mobility $\mu=10^{4} \mathrm{~cm}^{2} / \mathrm{Vs}$. In the following we will approximate $\sigma(q, \omega) \approx \sigma(q=0, \omega)$, which is usually valid for wave vectors that are small in the atomic scale set by Fermi wavelength or the corresponding screening length. However, if only a few Landau levels are occupied, the $q$ dependence becomes important already at the scale set by the magnetic length. ${ }^{41}$ Therefore, in the following we restrict the analysis to the long-wavelength limit, $q l_{B} \ll 1$. . $^{42,43}$

\section{B. Magnetoplasmon excitations for $E_{0}<\mu_{c}<E_{1}$}

We now focus on the case where the chemical potential, which can be tuned by voltages applied to external gates, is located between the $n=0$ and +1 Landau levels. At zero temperature $n_{F}\left(E_{n}\right)=0$ for $E_{n} \geqslant E_{1}$ and $n_{F}\left(E_{n}\right)=1$ for $E_{n} \leqslant E_{0}$. For $\omega \leqslant 2 \omega_{B}$ graphene conductivities can be approximated by using only the $n=0$ intraband term as shown in Figs. 1(a) and 1(b), and $\eta(0)$ and $\chi(0)$ are given by

$$
\begin{aligned}
& \eta(0)=q d \frac{\tilde{\omega}}{\omega} \frac{\omega_{B}^{2}}{\tilde{\omega}^{2}-\omega_{B}^{2}}, \\
& \chi(0)=q d \frac{\omega_{B}}{\omega} \frac{\omega_{B}^{2}}{\tilde{\omega}^{2}-\omega_{B}^{2}},
\end{aligned}
$$

where $\tilde{\omega}=\omega+i \tau^{-1}$, and the effective length $d$ is defined as $e^{2} /(\pi \hbar \epsilon d)=\omega_{B}$ (e.g., with $\mathrm{SiO}_{2}$ as the substrate $d \approx 20 \mathrm{~nm}$ $\approx 2.5 l_{B}$ at $B=10 \mathrm{~T}$, roughly equals to 80 times the graphene lattice constant). Equation (5) can be solved, and only keeping the positive frequencies we get

$$
\omega_{ \pm}=\frac{\sqrt{1+4 q d / 3} \pm 1}{2} \omega_{B},
$$

where the upper branch $\omega_{+}$is a bulklike mode and the lower branch $\omega_{-}$is the edge magnetoplasmon, corresponding to a gapless spectrum $\omega_{-} \rightarrow 0$ as $q \rightarrow 0$. The dispersion Eq. (10) differs from the result $\omega \sim q \log q,{ }^{25,44}$ obtained at 

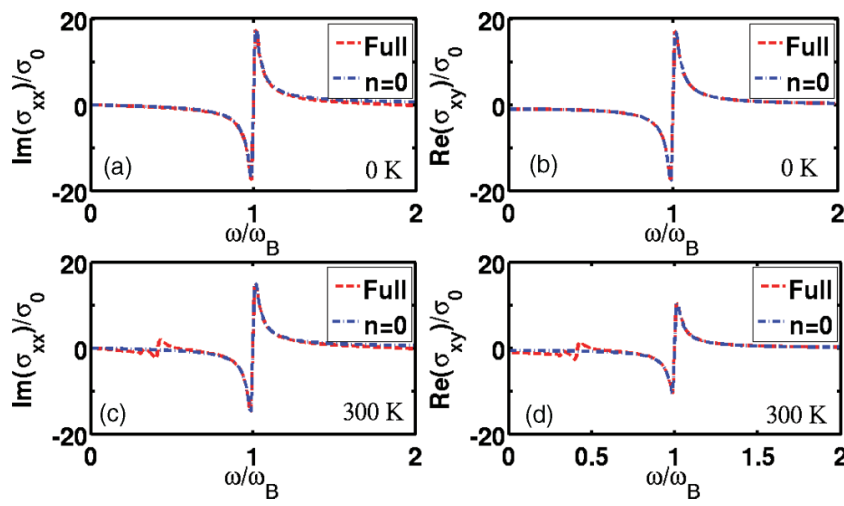

FIG. 1. (Color online) (a-d) The graphene conductivities $\operatorname{Im}\left(\sigma_{x x}\right)$ and $\operatorname{Re}\left(\sigma_{x y}\right)$ as functions of $\omega$ for chemical potential $\mu_{c}=0.5 \omega_{B}$ and $B=10 \mathrm{~T}$, at temperatures 0 and $300 \mathrm{~K}$ (corresponding to $\hbar \omega_{B} / 6$ ). Here $\sigma_{0}=2 e^{2} / h$. Red dashed lines are calculated from Eqs. (7a) and (7b), and blue dash-dotted lines are approximate estimations with only the $n=0$ term.

the long-wavelength and low-frequency limit in the quantum Hall regime where the Hall conductivity $\sigma_{x y}(\omega)$ is much larger than the diagonal conductivity $\sigma_{x x}(\omega)$.

At a nonzero temperature the Fermi function is no longer given by a step function, and several Landau levels are partially occupied. For $k_{B} T \ll \hbar \omega_{B}$ we can approximate $n_{F}\left(E_{0}\right)=$ $1-a(T)$ and $n_{F}\left(E_{1}\right)=b(T)$, and other levels remain either fully occupied or empty. As shown in Figs. 1(c) and 1(d), graphene conductivities can be well approximated by only keeping the $n=0$ term, and for frequencies close to $\omega_{B}$ the auxiliary functions are given by

$$
\begin{aligned}
\eta(T) & =[1-b(T)] \eta(0), \\
\chi(T) & =[1-b(T)-2 a(T)] \chi(0) .
\end{aligned}
$$

The small difference in the temperature dependencies of these functions, defined as $\delta(T)=1-[\chi(T) / \chi(0)] /$ $[\eta(T) / \eta(0)]=2 a(T) /[1-b(T)]$, results in a fundamental change in the edge magnetoplasmon properties. When $\tau \rightarrow$ $\infty$, the implicit dispersion relation now becomes

$$
3 \omega \frac{\omega^{2}-1}{\omega \pm[1-\delta(T)]}=q d[1-b(T)],
$$

where $\omega$ is in units of $\omega_{B}$. This third-order equation has three real solutions for $\omega$, corresponding to three plasmon branches. In the zero-temperature case, $a(T)=b(T)=0$, the dispersion relation given by Eq. (10) is reproduced. At a nonzero temperature, a third plasmon branch appears due to $\delta(T) \neq 0$. In ordinary two-dimensional semiconductor systems, the gap between the valence and conduction bands renders the interband transitions in Eqs. (7a) and (7b) inefficient at lower energies, and only the intraband terms contribute to the conductivity tensor. Furthermore, the hole states $E_{n}$ with $n<0$ continue to be fully occupied at low temperatures, so that the only temperature dependence in $\sigma_{x x}$ and $\sigma_{x y}$ arises from $\left[n_{F}\left(E_{0}\right)-n_{F}\left(E_{1}\right)\right]$ so that $\delta(T)=0$ and only two plasmon branches appear. In graphene, in contrast, due to the degeneracy of the $n=0$ electron and hole Landau levels, the thermal factor in $\sigma_{x x}$ becomes $\left[n_{F}\left(-E_{1}\right)-n_{F}\left(E_{1}\right)\right]$ and in $\sigma_{x y}$ the factor is $\left[2 n_{F}(0)-n_{F}\left(E_{1}\right)-n_{F}\left(-E_{1}\right)\right], \delta(T) \neq 0$, and a third plasmon branch appears.

The properties of the extra branch can be determined from the approximate dispersion relation given by Eq. (12). First, we see that there is only one solution propagating in the positive $("+")$ direction, having $\omega_{+}>1$. This is essentially the bulklike magnetoplasmon. There are two solutions propagating in the negative ("-") direction, one with $\omega_{-}<1-\delta(T)$ and the other with $\omega_{-}>1$. The former is the ordinary edge magnetoplasmon whose dispersion relation starts from $\omega(q=0)=0$, while the latter is the mode not seen in ordinary 2DESs. The new mode starts at $\omega(q=0)=1$ and has a linear dispersion with a velocity $v \approx \frac{1}{3} a(T) \omega_{B} d$ [here $v=\partial \omega / \partial q$ and is calculated by taking the derivatives of Eq. (12) at $\omega \rightarrow 1$ ], which is determined primarily by $a(T)$. Hence, its dispersion can be controlled by temperature and chemical potential. We would also like to point out that the absence of solutions in the negative branch for $1-\delta(T)<$ $\omega_{-}<1$ is suggestive of a gap appearing in the plasmonic spectrum; however, since our analysis only uses the small- $q$ limit of the conductivity tensor, this result is not conclusive.

We now proceed to verify the above predictions with a numerical solution of the integral equation with the full kernel. Combining Eqs. (1) and (2), the potential can be self-consistently solved. We expand the in-plane potential as a Laguerre series: ${ }^{33,34}$

$$
\Phi(x)=\exp (q x) \sum_{n=0}^{\infty} c_{n} L_{n}(-2 q x) .
$$

Using the orthonormality of the Laguerre polynomials, we obtain

$$
\frac{c_{m}}{2}=\sum_{n=0}^{\infty}\left[\eta J_{m n}+\eta(2 n+1) I_{m} \pm \chi I_{m}\right] c_{n},
$$
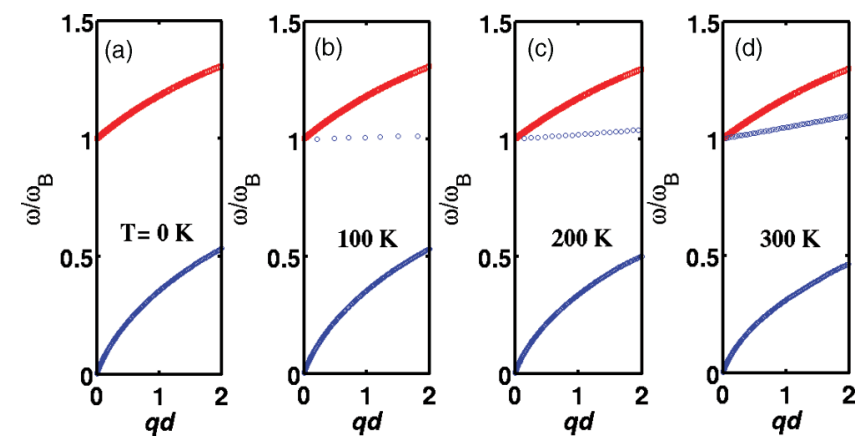

FIG. 2. (Color online) (a-d) The edge magnetoplasmon dispersions obtained by a numerical solution of the full integral equation at different temperatures for $B=10 \mathrm{~T}\left(\hbar \omega_{B}=1800 \mathrm{~K}\right)$ and $\mu_{c}=0.5 \hbar \omega_{B}$. The higher-frequency (red squared) branch starts from $\omega_{B}$ and the lower (blue circular) branch starts from zero. For higher temperatures (b-d), an extra branch appears at $\omega \gg \omega_{B}$. It is clearly seen that the velocity of the new branch increases with temperature while the other two branches are largely independent of temperature. 

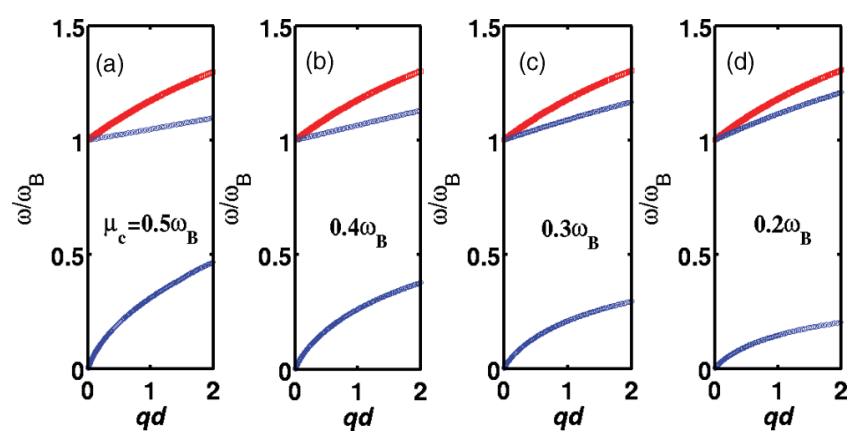

FIG. 3. (Color online) (a-d) The edge magnetoplasmon dispersions obtained by a numerical solution of the full integral equation at chemical potentials $\mu_{c}=0.5 \hbar \omega_{B}, 0.4 \hbar \omega_{B}, 0.3 \hbar \omega_{B}$, and $0.2 \hbar \omega_{B}$ for $B=10 \mathrm{~T}$ and temperature $300 \mathrm{~K}\left(\approx \hbar \omega_{B} / 6\right)$. For a decreasing chemical potential, the extra branch is seen to have a larger dispersion, while that of the low-frequency branch is reduced.

with

$$
\begin{aligned}
J_{m n}= & \int_{-\infty}^{0} d y \exp (y) L_{m}(-2 y) \int_{-\infty}^{0} d y^{\prime} L_{q}\left(y-y^{\prime}\right) \\
& \times\left[1-\frac{\partial^{2}}{\partial y^{\prime 2}}\right] \exp \left(y^{\prime}\right) L_{n}\left(-2 y^{\prime}\right), \\
I_{m}= & \int_{-\infty}^{0} d y \exp (y) L_{m}(-2 y) L_{q}(y),
\end{aligned}
$$

where $y=q x$ and $y^{\prime}=q x^{\prime}$ are the integration variables. Equation (14) is a standard eigenvalue equation and can be numerically solved to find the appropriate physical solutions.

At zero temperature, i.e., $\delta(T)=0$, the dispersion is very similar to that in an ordinary 2DES. Numerical results using the full expressions for the conductivities and the kernel verify this as shown in Fig. 2. However, as the temperature rises, an extra branch around $\omega \approx \omega_{B}$ gradually arises, seen in Figs. 2(b)2 (d). At room temperature, $300 \mathrm{~K}\left(\approx \hbar \omega_{B} / 6\right)$, this branch is clearly visible (a broadband), and should be possible to detect experimentally. The width of the extra branch also can be tuned by varying the chemical potential as shown in Fig. 3, and the linear dispersions can be found at very-large-energy ranges. All these numerical results have been well predicted by the approximate analysis.

\section{CONCLUSIONS}

In conclusion, we have shown that the edge magnetoplasmons in graphene exhibit an extra energy branch due to the temperature effects. The dispersion of this branch is linear at the $q \rightarrow 0$ limit, and the bandwidth can be tuned by temperature, chemical potential, or magnetic field. This extra branch reflects the novel property of the $n=0$ Landau level (electron-hole degenerate Landau level) in graphene, which has not been demonstrated before.

\section{ACKNOWLEDGMENT}

This work was funded by the Swedish Research Council (VR). *weihua.wang@chalmers.se.

${ }^{1}$ A. K. Geim and K. S. Novoselov, Nat. Mater. 6, 183 (2007).

${ }^{2}$ A. H. Castro Neto, F. Guinea, N. M. R. Peres, K. S. Novoselov, and A. K. Geim, Rev. Mod. Phys. 81, 109 (2009).

${ }^{3}$ S. Das Sarma, S. Adam, E. H. Hwang, and E. Rossi, Rev. Mod. Phys. 83, 407 (2011).

${ }^{4}$ R. R. Nair, P. Blake, A. N. Grigorenko, K. S. Novoselov, T. J. Booth, T. Stauber, N. M. R. Peres, and A. K. Geim, Science 320, 1308 (2008).

${ }^{5}$ S. A. Mikhailov and K. Ziegler, Phys. Rev. Lett. 99, 016803 (2007). ${ }^{6}$ G. W. Hanson, J. Appl. Phys. 103, 064302 (2008).

${ }^{7}$ Q. Bao, H. Zhang, B. Wang, Z. Ni, C. H. Y. X. Lim, Y. Wang, D. Y. Tang, and K. P. Loh, Nature Photon. 5, 411 (2011).

${ }^{8}$ M. Liu, X. Yin, E. Ulin-Avila, B. Geng, T. Zentgraf, L. Ju, F. Wang, and X. Zhang, Nature (London) 474, 64 (2011).

${ }^{9}$ A. Vakil and N. Engheta, Science 332, 1291 (2011).

${ }^{10}$ W. Zhou, J. Lee, J. Nanda, S. T. Pantelides, S. J. Pennycook, and J.-C. Idrobo, Nature Nanotechnol. 7, 161 (2012).

${ }^{11}$ E. G. Mishchenko, A. V. Shytov, and P. G. Silvestrov, Phys. Rev. Lett. 104, 156806 (2010).

${ }^{12}$ A. Y. Nikitin, F. Guinea, F. J. García-Vidal, and L. Martín-Moreno, Phys. Rev. B 84, 161407 (2011).

${ }^{13}$ F. H. L. Koppens, D. E. Chang, and F. J. García de Abajo, Nano Lett. 11, 3370 (2011).

${ }^{14}$ M. Jablan, H. Buljan, and M. Soljačić, Phys. Rev. B 80, 245435 (2009).
${ }^{15}$ A. Y. Nikitin, F. Guinea, F. J. Garcia-Vidal, and L. Martin-Moreno, Phys. Rev. B 85, 081405 (2012).

${ }^{16} \mathrm{~S}$. Thongrattanasiri, F. H. L. Koppens, and F. J. García de Abajo, Phys. Rev. Lett. 108, 047401 (2012).

${ }^{17}$ H. Yan, X. Li, B. Chandra, G. Tulevski, Y. Wu, M. Freitag, W. Zhu, P. Avouris, and F. Xia, Nature Nanotechnol. 7, 330 (2012).

${ }^{18}$ F. Rana, IEEE Trans. Nano Technol. 7, 91 (2008).

${ }^{19}$ D. D. Dubinov, V. Ya Aleshkin, V. Mitin, T. Otsuji, and V. Ryzhii, J. Phys.: Condens. Matter 23, 145302 (2011).

${ }^{20}$ D. B. Mast, A. J. Dahm, and A. L. Fetter, Phys. Rev. Lett. 54, 1706 (1985).

${ }^{21}$ J. W. Wu, P. Hawrylak, and J. J. Quinn, Phys. Rev. Lett. 55, 879 (1985).

${ }^{22}$ A. L. Fetter, Phys. Rev. B 32, 7676 (1985).

${ }^{23}$ A. L. Fetter, Phys. Rev. B 33, 3717 (1986).

${ }^{24}$ D. C. Glattli, E. Y. Andrei, G. Deville, J. Poitrenaud, and F. I. B. Williams, Phys. Rev. Lett. 54, 1710 (1985).

${ }^{25}$ V. A. Volkov and S. A. Mikhailov, Sov. Phys. JETP 67, 1639 (1988).

${ }^{26}$ I. L. Aleiner and L. I. Glazman, Phys. Rev. Lett. 72, 2935 (1994).

${ }^{27}$ O. G. Balev and P. Vasilopoulos, Phys. Rev. Lett. 81, 1481 (1998).

${ }^{28}$ M. Wassermeier, J. Oshinowo, J. P. Kotthaus, A. H. MacDonald, C. T. Foxon, and J. J. Harris, Phys. Rev. B 41, 10287 (1990).

${ }^{29}$ O. G. Balev, P. Vasilopoulos, and H. O. Frota, Phys. Rev. B 84, 245406 (2011)

${ }^{30}$ O. G. Balev, A. C. A. Ramos, and H. O. Frota, Phys. Rev. B 85, 205421 (2012) 
${ }^{31}$ H. Yan, Z. Li, X. Li, W. Zhu, P. Avouris, and F. Xia, arXiv: $1204.4398 \mathrm{v} 1$

${ }^{32}$ I. Crassee, M. Orlita, M. Potemski, A. L. Walter, M. Ostler, T. Seyller, I. Gaponenko, J. Chen, and A. B. Kuzmenko, Nano Lett. 12, 2470 (2012).

${ }^{33}$ J.-W. Wu, P. Hawrylak, G. Eliasson, and J. J. Quinn, Phys. Rev. B 33, 7091 (1986).

${ }^{34}$ W. Wang, P. Apell, and J. Kinaret, Phys. Rev. B 84, 085423 (2011).

${ }^{35}$ The full 3D potential is given by $\Phi(x, y, z, t)=$ $e^{i(q y-\omega t)} \frac{1}{\epsilon} \int_{-\infty}^{0} d x^{\prime} \int_{-\infty}^{\infty} \frac{d k}{4 \pi} \frac{e^{\left(k^{2}+q^{2}\right)^{1 / 2} z}}{\left(k^{2}+q^{2}\right)^{1 / 2}} e^{i k\left(x-x^{\prime}\right)} \rho\left(x^{\prime}\right)$.

${ }^{36}$ O. L. Berman, G. Gumbs, and Y. E. Lozovik, Phys. Rev. B 78, 085401 (2008).
${ }^{37}$ R. Roldàn, J.-N. Fuchs, and M. O. Goerbig, Phys. Rev. B 80, 085408 (2009).

${ }^{38}$ J.-Y. Wu, S.-C. Chen, O. Roslyak, G. Gumbs, and M.-F. Lin, ACS Nano 5, 1026 (2011).

${ }^{39}$ Y. Zheng and T. Ando, Phys. Rev. B 65, 245420 (2002).

${ }^{40}$ V. P. Gusynin, S. G. Sharapov, and J. P. Carbotte, J. Phys.: Condens. Matter 19, 026222 (2007).

${ }^{41} \mathrm{G}$. Giuliani and G. Vignale, Quantum Theory of the Electron Liquid (Cambridge University Press, Cambridge, 2005).

${ }^{42}$ O. G. Balev and P. Vasilopoulos, Phys. Rev. B 56, 13252 (1997).

${ }^{43}$ O. G. Balev and P. Vasilopoulos, Phys. Rev. B 59, 2807 (1999).

${ }^{44}$ V. A. Volkov and S. A. Mikhailov, JETP Lett. 42, 556 (1985). 